\title{
ARTICLE
}

\section{Large-scale quantum-emitter arrays in atomically thin semiconductors}

\author{
Carmen Palacios-Berraquero ${ }^{1, \star}$, Dhiren M. Kara ${ }^{1, \star}$, Alejandro R.-P. Montblanch ${ }^{1}$, Matteo Barbone ${ }^{1,2}$,
} Pawel Latawiec ${ }^{3}$, Duhee Yoon ${ }^{2}$, Anna K. Ott ${ }^{2}$, Marko Loncar $^{3}$, Andrea C. Ferrari ${ }^{2} \&$ Mete Atatüre $^{1}$

Quantum light emitters have been observed in atomically thin layers of transition metal dichalcogenides. However, they are found at random locations within the host material and usually in low densities, hindering experiments aiming to investigate this new class of emitters. Here, we create deterministic arrays of hundreds of quantum emitters in tungsten diselenide and tungsten disulphide monolayers, emitting across a range of wavelengths in the visible spectrum $(610-680 \mathrm{~nm}$ and $740-820 \mathrm{~nm})$, with a greater spectral stability than their randomly occurring counterparts. This is achieved by depositing monolayers onto silica substrates nanopatterned with arrays of 150-nm-diameter pillars ranging from 60 to $190 \mathrm{~nm}$ in height. The nanopillars create localized deformations in the material resulting in the quantum confinement of excitons. Our method may enable the placement of emitters in photonic structures such as optical waveguides in a scalable way, where precise and accurate positioning is paramount.

\footnotetext{
${ }^{1}$ Cavendish Laboratory, University of Cambridge, JJ Thomson Avenue, Cambridge CB3 OHE, UK. ${ }^{2}$ Cambridge Graphene Centre, University of Cambridge, Cambridge CB3 OFA, UK. ${ }^{3}$ John A. Paulson School of Engineering and Applied Science, Harvard University, 29 Oxford Street, Cambridge, Massachusetts 02138, USA. * These authors contributed equally to this work. Correspondence and requests for materials should be addressed to A.C.F. (email: acf26@cam.ac.uk) or to M.A. (email: ma424@cam.ac.uk).
} 
T ransition metal dichalcogenides (TMDs) are optically active, semiconducting layered materials (LMs) which can be exfoliated down to monolayers. These are of particular interest, as they exhibit properties such as an optically accessible valley degree of freedom that is locked to the exciton $\operatorname{spin}^{1-3}$, strong two-dimensional confinement, favouring bound excitonic states ${ }^{4,5}$ and the opportunity to investigate many-body physics $^{6}$. Intrinsic properties of LMs-atomically precise interfaces, lack of dangling bonds, flexibility and the possibility of stacking different LMs into functional heterostructures ${ }^{7,8}$ make them not only interesting for fundamental physics but also suitable for technological applications ${ }^{9,10}$. For these reasons, the identification of quantum emitters (QEs) in $\mathrm{LMs}^{11-16}$ has generated much excitement in the field of two-dimensional nanophotonics $^{10,17-19}$ and quantum technologies ${ }^{9,20}$.

Single photon emission has been seen from QEs in tungsten diselenide ( $\mathrm{WSe}_{2}$ ) with both above bandgap ${ }^{11-16}$ and resonant optical excitation ${ }^{21}$. In addition, QEs in $\mathrm{WSe}_{2}$ and tungsten disulphide $\left(\mathrm{WS}_{2}\right)$ have been implemented in heterostructures to achieve electrically driven single-photon emission ${ }^{22}$. However, the origin of QEs in TMDs is still unclear, and has been assigned to both defects ${ }^{11-16}$ and strain gradients ${ }^{21,23,24}$. Experiments on these QEs have, until now, been reliant on their rare and random occurrence. Deterministic creation of precisely positioned LM QEs in large numbers is important for accelerating the study of these emitters, as well as opening up the prospect for scalability and on-chip applications.

Here, we report a method to create arrays of single-photon emitting QEs in $\mathrm{WSe}_{2}$ and quantum-like emitters in $\mathrm{WS}_{2}$ using a nanopatterned silica substrate. We obtain structures with QE numbers typically in the range of hundreds. The quality of these deterministic QEs surpasses that of their randomly appearing counterparts, with spectral wanderings of $\sim 0.1 \mathrm{meV}$ - an order of magnitude lower than previous reports ${ }^{11-15}$. Our technique is a crucial first step towards solving the scalability challenge for LM-based quantum photonic devices.

\section{Results}

Nanopatterned substrate preparation and characterization. To create large-scale QE arrays in LMs, we place the active material on patterned structures fabricated on the substrate in order to create spatially localized physical disturbances to the otherwise flat LM flakes. To this end, we first pattern arrays of nanopillars of different heights, ranging from 60 to $190 \mathrm{~nm}$, on silica substrates using electron beam lithography. Figure 1a shows a scanning electron microscope image of one such substrate of $130 \mathrm{~nm}$ nanopillar height. We place layers of $\mathrm{WSe}_{2}$ and $\mathrm{WS}_{2}$ on the nanopillars as follows. Bulk $\mathrm{WSe}_{2}$ and $\mathrm{WS}_{2}$ crystals are characterized before exfoliation as described in ref. 22. These are then exfoliated on a polydimethylsiloxane layer by micromechanical cleavage $\mathrm{e}^{7,25}$. Single-layer (1L) samples are identified first by optical contrast ${ }^{26}$, and the selected $1 \mathrm{~L}-\mathrm{WSe} \mathrm{S}_{2}$ and $1 \mathrm{~L}-\mathrm{WS}_{2}$ flakes are then placed onto the patterned nanopillar substrate via an all-dry viscoelastic transfer technique due to their higher adhesion to $\mathrm{SiO}_{2}$ (refs 7,19,27), as schematically shown in Fig. 1b. After exfoliation and transfer, the $1 \mathrm{~L}-\mathrm{WS} \mathrm{e}_{2}$ and $1 \mathrm{~L}-\mathrm{WS}_{2}$ flakes are characterized by Raman spectroscopy ${ }^{28,29}$, photoluminescence (PL) ${ }^{30}$ and atomic force microscopy (AFM), confirming the transfer and that the process does not damage the samples (see Supplementary Fig. 1 and Supplementary Note 1 for the corresponding spectra and discussion). Figure 1c is an AFM scan of a $1 \mathrm{~L}-\mathrm{WSe}_{2}$ flake over a single nanopillar. The bottom panel of Fig. 1c plots the height profile of the $1 \mathrm{~L}-\mathrm{WSe} \mathrm{e}_{2}$ flake taken along the dashed pink line. This reveals how the flake (solid pink line) tents over the nanopillar. The blue-shaded area corresponds to the measured profile of a bare nanopillar. Figure $1 \mathrm{~d}$ is a darkfield optical microscopy image of part of a $43,000 \mu \mathrm{m}^{2} 1 \mathrm{~L}-\mathrm{WSe}_{2}$ flake on a substrate patterned with a $4-\mu \mathrm{m}$-spaced nanopillar array with nominal height of $130 \mathrm{~nm}$. The regularly spaced bright spots correspond to nanopillar sites. We see locations providing brighter scattering (two examples are encircled in pink) and others showing fainter intensity (two examples are encircled in blue). By correlating with AFM measurements we find that the former correspond to locations where the $1 \mathrm{~L}-\mathrm{WSe} \mathrm{e}_{2}$ tents over the nanopillars and the latter correspond to locations where the flake is pierced by the nanopillars (see Supplementary Fig. 2). On average, we find that two-third of the sites are not pierced during the deposition step.

Quantum light from $\mathrm{WSe}_{2}$-based deterministic QEs. Figure 2a is an integrated raster scan map of PL emission at $\sim 10 \mathrm{~K}$ of six adjacent non-pierced nanopillar sites in the region enclosed by the green dashed line in Fig. 1d. The most prominent feature is the $\sim \times 10$ increase in intensity at the location of every nanopillar. Figure $2 \mathrm{~b}$ reveals the source of this emission intensity enhancement: spectra taken at each nanopillar location display bright sub-nanometre linewidth emission peaks. Figure $2 \mathrm{c}$ demonstrates the single-photon nature of this emission via photon-correlation measurements taken (from left to right) at the first, third and fourth nanopillar locations. Ten nanometre bandpass filters, indicated by the pink, green and blue highlighted areas in the panels of Fig. $2 \mathrm{~b}$, select the spectral windows for the photon-correlation measurements. We obtain $g^{(2)}(0)$ values of $0.0868 \pm 0.0645,0.170 \pm 0.021$ and $0.182 \pm 0.028$, respectively, uncorrected for background emission or detector response. While these surpass those in early reports ${ }^{11-15}$, we expect the quality of the single-photon emission from the QEs to improve under resonant excitation ${ }^{31}$. Out of the 53 unpierced nanopillar sites in this substrate we find sub-nm emission peaks in 51 of them, giving $\sim 96 \%$ yield in QE generation. Their emission wavelength ranges between 730 and $820 \mathrm{~nm}$ (see Supplementary Fig. 3 for statistics), equivalent to a redshift distributed between 50 and $280 \mathrm{meV}$ from the unbound exciton emission energy at $\sim 1.755 \mathrm{eV}$ (ref. 32), as observed for the naturally occurring QEs in $\mathrm{WSe}_{2}$ (refs 11-15). The fine-structure splitting $(200-730 \mu \mathrm{eV})$ and the emission linewidths as narrow as $\sim 180 \mu \mathrm{eV}(\sim 0.08 \mathrm{~nm})$ are also consistent with previous reports $^{11-15}$ (Supplementary Fig. 3) advocating that these deterministically created QEs are of the same nature as the randomly appearing ones.

Effect of nanopillar height on WSe $\mathbf{H}_{2}$-based QEs. To study the effect of nanopillar height, we carry out similar optical measurements of $1 \mathrm{~L}-\mathrm{WSe}_{2}$ flakes deposited on nanopillars of height $\sim 60$ and $\sim 190 \mathrm{~nm}$. The spectra taken at the $60-\mathrm{nm}$ nanopillars have multiple peaks of $\sim 1 \mathrm{~nm}$ linewidth on average (see Supplementary Fig. 3 for example spectra). In contrast, Fig. $2 \mathrm{~d}$ is a representative spectrum taken from the $190 \mathrm{~nm}$ nanopillars, displaying a better isolated, single sub-nm emission peak. The inset reveals a $722 \mu \mathrm{eV}$ fine-structure splitting for this QE. We do not see clear nanopillar height dependence in the emission wavelength and fine-structure splitting (see Supplementary Fig. 3 for statistics). Although we often observe multiple narrow emission lines from nanopillar locations, increasing the nanopillar height reduces the spread in the number of peaks arising at each. We verify this trend in Fig. 2e, a histogram of the probability that a given number of sub-nm emission peaks appear per nanopillar, for the different nanopillar heights (60, 130 and $190 \mathrm{~nm}$ in white, blue and purple, respectively). The likelihood of creating a single QE grows as nanopillar height is 
a

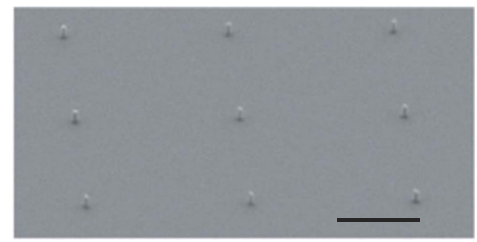

$\mathbf{b}_{1}$

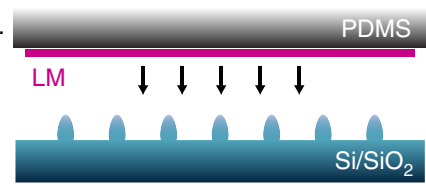

2 .
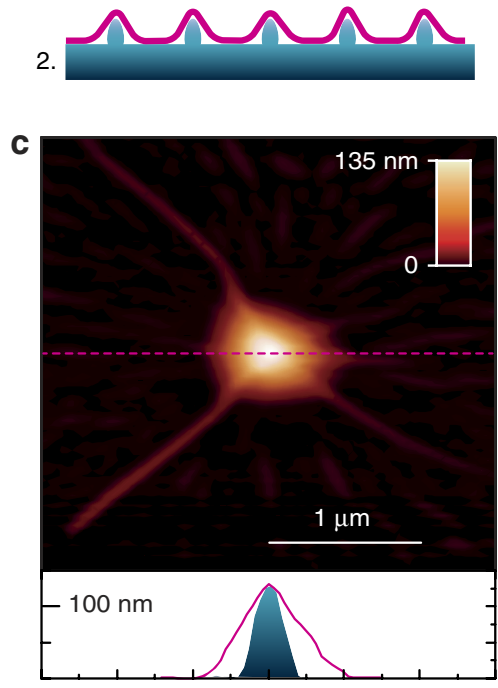

d

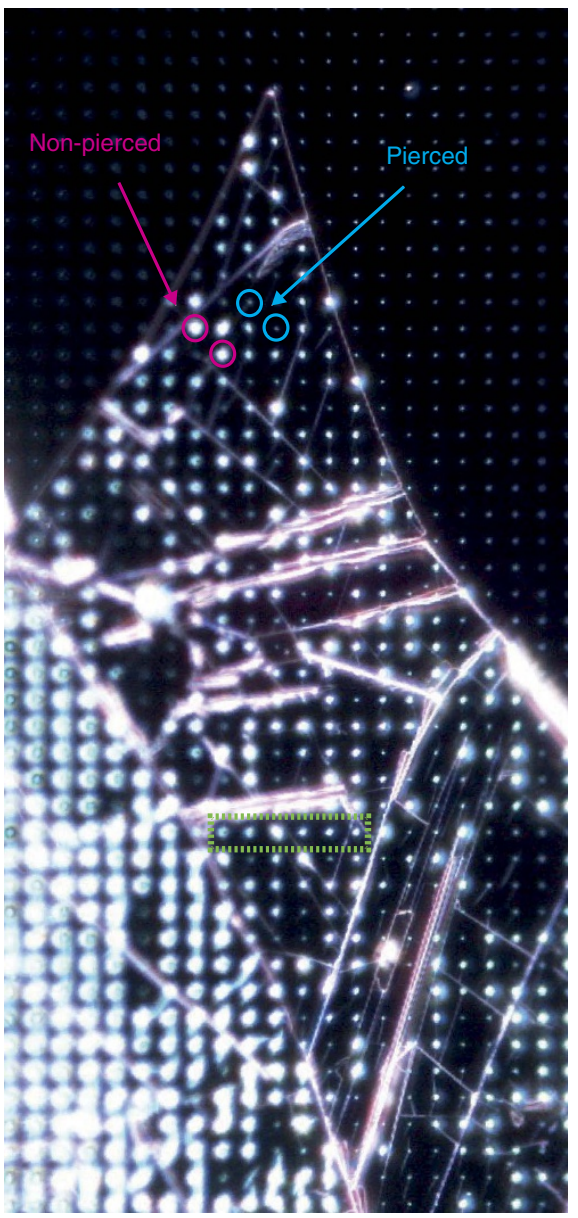

Figure 1 | Fabrication and characterization of scalable quantum confinement arrays. (a) SEM image of nanopillar substrate, fabricated by electron beam lithography. Black scale bar, $2 \mu \mathrm{m}$. (b) Illustration of the fabrication method: (1) mechanical exfoliation of LM on PDMS and all-dry viscoelastic deposition on patterned substrate; and (2) deposited LM on patterned substrate. (c, top) An AFM scan of $1 \mathrm{~L}-\mathrm{WS} \mathrm{e}_{2}$ on a nanopillar. (bottom) The AFM height profile of a bare nanopillar (blue-shaded region) and of the flake deposited over it (pink line), measured along the dashed pink line cut in the top panel. Colour-scale bar represents height in $\mathrm{nm}$ and white scale bar $1 \mu \mathrm{m}$. (d) Dark field optical microscopy image (real colour) of $1 \mathrm{~L}-\mathrm{WS} \mathrm{e}_{2}$ on nanopillar substrate (130 nm high, $4 \mu \mathrm{m}$ separation). The full image corresponds to a $170 \mu \mathrm{m}$ by $210 \mu \mathrm{m}$ area. The green box highlights six adjacent nanopillars within the $1 \mathrm{~L}-\mathrm{WS} \mathrm{e}_{2}$ region, measured in Fig. 2. The blue circles indicate two pierced nanopillars, and the pink circles indicate two non-pierced nanopillars. PDMS, polydimethylsiloxane; SEM, scanning electron microscope.

increased. For the $190 \mathrm{~nm}$ nanopillars, 50\% of all nanopillar sites host a single QE with one emission peak, as indicated by the purple bars. Spectral wandering of the peaks as a function of time also displays a strong dependence on the nanopillar height. To quantify this dependence, we record the maximum range of emission wavelength wandering per $\mathrm{QE}$ over tens of seconds. The solid black circles in Fig. $2 \mathrm{f}$ correspond to the mean of these values for each group of QEs pertaining to each nanopillar height, for 17 different QEs in total, with the error bars displaying the standard deviation of these distributions. We observe a reduction from a few $\mathrm{meV}$ for $60 \mathrm{~nm}$ nanopillars to below $0.25 \mathrm{meV}$ (average) for the tallest $190 \mathrm{~nm}$ nanopillars (see Supplementary Fig. 4), reaching as low as $0.1 \mathrm{meV}$. To the best of our knowledge, this is the lowest spectral wandering seen to date in LM QEs ${ }^{11-15}$. Hence, these deterministic QEs are comparable, and even superior, in spectral stability to their randomly appearing counterparts. The dependence of certain QE characteristics on nanopillar height, along with shifts in the delocalized neutral exciton peak $\left(\mathrm{X}^{0}\right)$ at room temperature ${ }^{30}$ at the nanopillar locations (see Supplementary Fig. 5), suggest that a localized strain gradient induced by the nanopillars might be playing an active role in producing QEs, as well as determining their specific optical properties ${ }^{21,23,24}$.

Deterministic QE creation in $\mathrm{WS}_{\mathbf{2}}$ monolayers. The method we present for QE creation is not restricted to a specific LM. We predict a similar effect on different LMs and test this by using $1 \mathrm{~L}-\mathrm{WS}_{2}$. Despite previous efforts to measure QEs in $1 \mathrm{~L}-\mathrm{WS}_{2}$, there has only been one previous report of single-photon emission in this material ${ }^{22}$. Figure 3 a shows an integrated PL intensity raster scan map taken at $\sim 10 \mathrm{~K}$ of a $1 \mathrm{~L}-\mathrm{WS}_{2}$ on a substrate with 170-nm-high nanopillars square array spaced by $3 \mu \mathrm{m}$. The inset shows a true-colour dark-field optical microscopy image of the same flake, where the red areas (due to fluorescence) are $1 \mathrm{~L}-\mathrm{WS}_{2}$. Once again, the brighter spots correspond to the unpierced nanopillar locations, as verified by AFM measurements, and show overlap with the bright fluorescence spots in the PL intensity image where, similar to $\mathrm{WSe}_{2}$, intensity is increased (here by a factor $\sim 4$ ) at every one of the 22 non-pierced nanopillar sites in the flake. Panel 1 of Fig. $3 \mathrm{~b}$ shows the typical $1 \mathrm{~L}-\mathrm{WS}_{2}$ emission spectrum at $\sim 10 \mathrm{~K}$ (ref. 22), measured from a flat region of the same flake away from the nanopillars. The $\mathrm{X}^{0}$ and $\mathrm{X}^{-}$unbound 
a

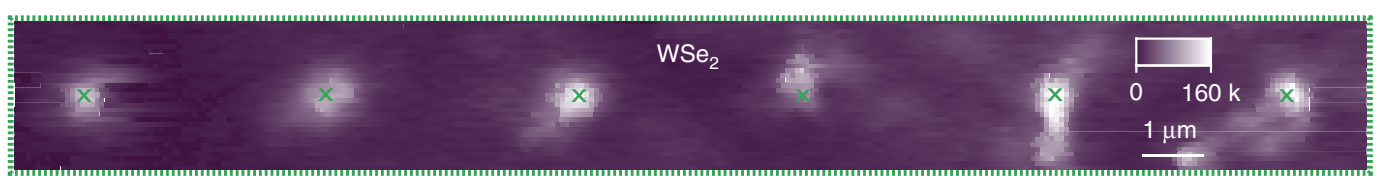

b

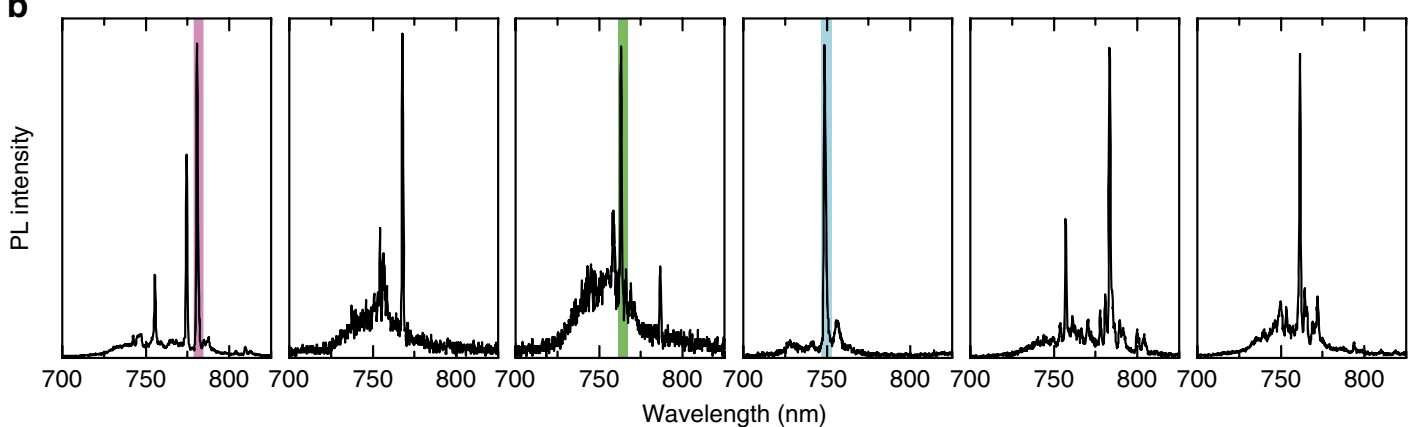

C

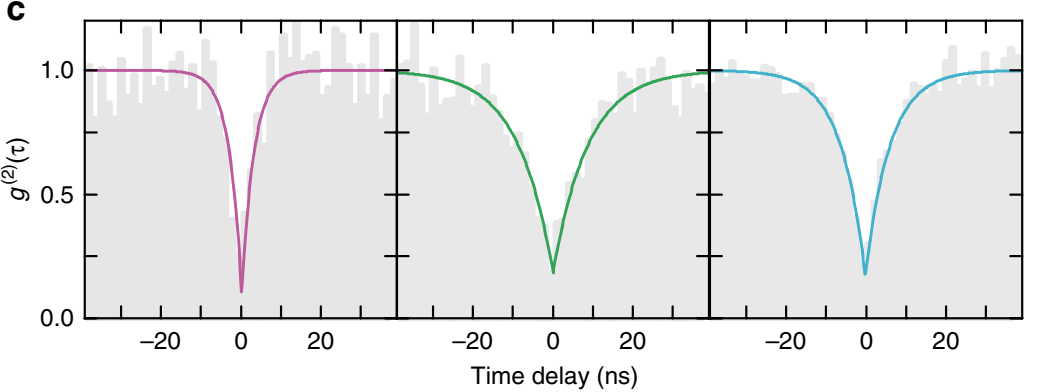

d

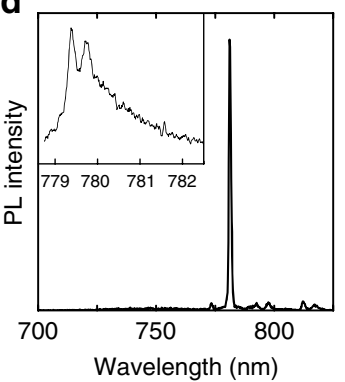

e
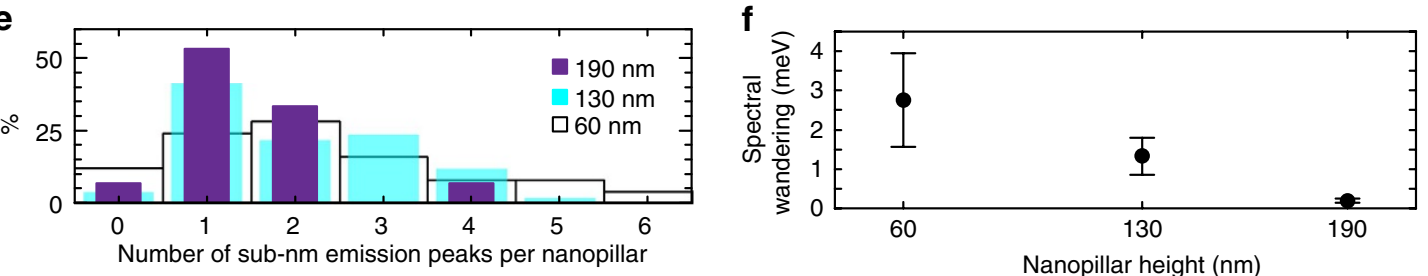

Figure 2 | Creation of quantum emitter arrays in 1L-WSe $\mathbf{2}_{\mathbf{2}}$ (a) Integrated PL intensity raster scan of the region enclosed by the green rectangle in Fig. 1d,

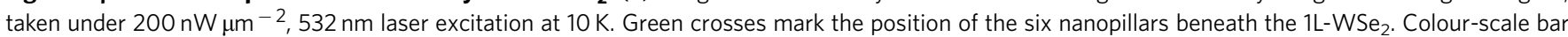
maximum, 160 kcounts s $^{-1}$. (b) PL spectra taken at each of the corresponding green crosses in $\mathbf{a}$, from left to right respectively, showing the presence of narrow lines at each nanopillar location. (c) Photon correlation measurements corresponding to the filtered spectral regions (10 nm wide) enclosed by the blue, green and pink rectangles, in $\mathbf{b}$, with $g^{(2)}(0)=0.087 \pm 0.065,0.17 \pm 0.02$ and $0.18 \pm 0.03$, and rise times of $8.81 \pm 0.80 \mathrm{~ns}, 6.15 \pm 0.36 \mathrm{~ns}$ and $3.08 \pm 0.41 \mathrm{~ns}$, respectively. (d) Spectrum taken from a $1 \mathrm{~L}-\mathrm{WSe}_{2}$ on a $190 \mathrm{~nm}$ nanopillar, showing lower background and a single sub-nm emission peak. Higher-resolution spectrum in the inset reveals the fine-structure splitting of this QE peak. An asymmetry can be seen in the spectrum, which has been previously attributed to a phonon sideband in naturally occurring $\mathrm{QEs}^{31}$. (e) Probability distribution (in \%) of the number of emission lines per nanopillar for samples using different nanopillar heights $(60,130$ and $190 \mathrm{~nm}$ in white, blue and purple, respectively). A trend of higher probability of single QE emission peaks per nanopillar location with increasing height is evident, reaching $50 \%$ for $190 \mathrm{~nm}$ nanopillars. (f) Increasing nanopillar height also leads to a reduction of spectral wandering. Solid black circles represent the mean value of spectral wandering of several QEs for a given nanopillar height, while the error bars represent the standard deviation of each distribution, both extracted from time-resolved high-resolution spectral measurements (Supplementary Fig. 4). A total number of seven samples was used to collect the statistics necessary for Fig. 2e,f.

excitons are labelled in the figure, while the broad red-shifted emission band arises from weakly localized or defect-related excitons in the $1 \mathrm{~L}-\mathrm{WS}_{2}$ at low temperatures ${ }^{22}$, and is present in this material regardless of location. Panels 2 and 3 of Fig. $3 \mathrm{~b}$ show representative PL emission spectra taken at nanopillars of heights $\sim 170$ and $\sim 190 \mathrm{~nm}$, respectively, where once again sub-nm spectral features arise. We note that we observe fine-structure splitting for $\mathrm{WS}_{2}$ in these QEs, which range from 300 to $810 \mu \mathrm{eV}$ (Supplementary Fig. 6), as represented in the panel insets corresponding to the spectral regions highlighted in red. We also measure the spectrum of several $\mathrm{WS}_{2}$ QEs as a function of time (see Supplementary Fig. 6) and find all spectral wandering values below $0.5 \mathrm{meV}$ over $1-2 \mathrm{~min}$. Figure $3 \mathrm{c}$ shows statistics on $\mathrm{QE}$ emission wavelength collected for over $\sim 80 \mathrm{QEs}$ for $1 \mathrm{~L}-\mathrm{WS}_{2}$ on 170-nm (white bars) and 190-nm (red bars) nanopillars. The wavelength distribution of the sub-nm emission lines, typically in the $610-680 \mathrm{~nm}$ region $\left(53-300 \mathrm{meV} \text { redshift from } \mathrm{X}^{0}\right)^{22}$, is as narrow as $\sim 20 \mathrm{~nm}$ for the $190-\mathrm{nm}$ nanopillars. Most nanopillar sites on $\mathrm{WS}_{2}$ show multiple sub-nm lines, suggesting the creation of several QEs at each site for these nanopillar heights. Figure 3d plots a histogram of the number of sub-nm peaks appearing at each nanopillar for both nanopillar heights. The trend is similar to that seen in $\mathrm{WSe}_{2}$, where higher nanopillars lead to a narrower spread in the number of peaks towards a higher likelihood of 
a
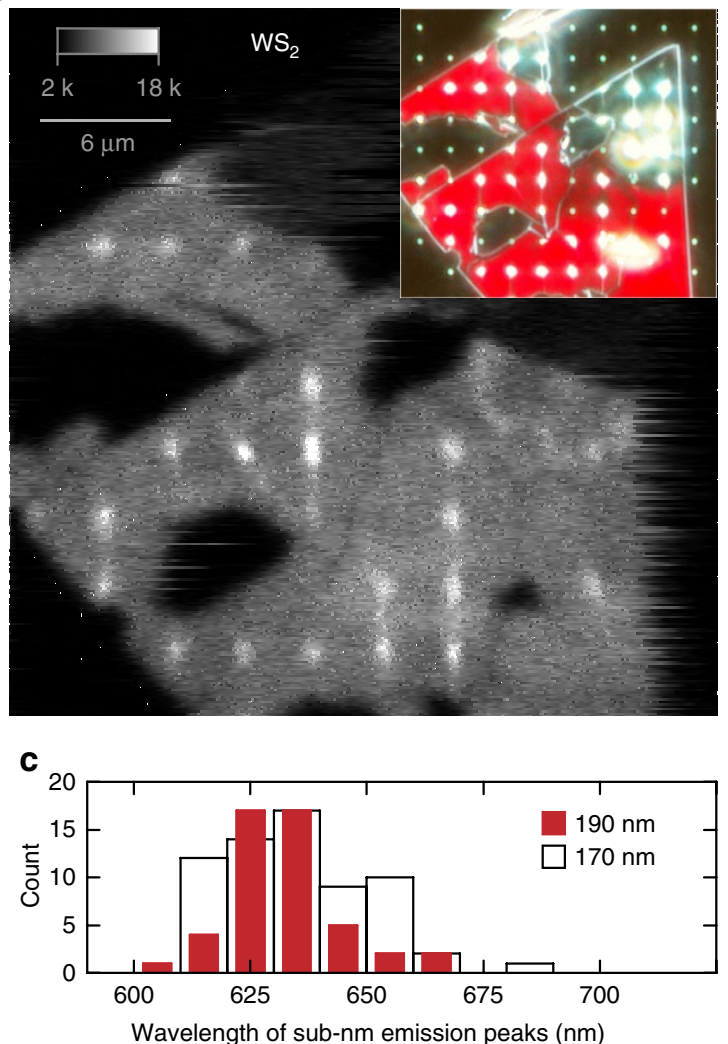

b

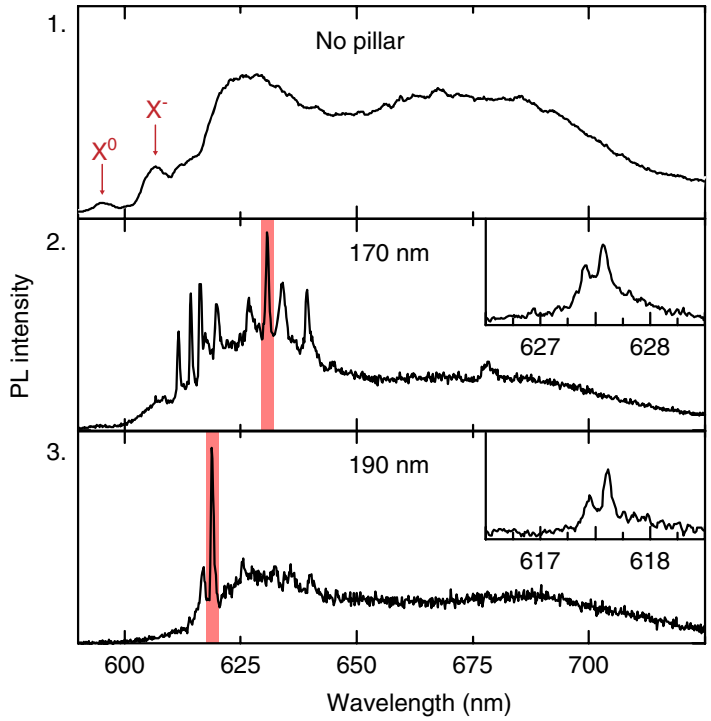

d

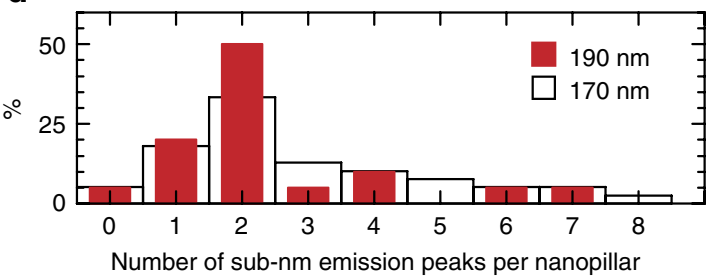

Figure 3 | Creation of quantum-emitter arrays in 1L-WS $\mathbf{W}_{\mathbf{2}}$ (a) Integrated PL intensity raster scan of a $1 \mathrm{~L}-\mathrm{WS}_{2}$ flake deposited on top of a $3 \mu \mathrm{m}$ spaced, 170-nm-high nanopillar array, taken at $300 \mathrm{nW} \mu \mathrm{m}^{-2}, 532 \mathrm{~nm}$ laser excitation at $10 \mathrm{~K}$. Colour-scale bar maximum is $18 \mathrm{kcounts} \mathrm{s}^{-1}$. Inset: true-colour DFM image of the same area. The red region corresponds to the $\mathrm{WS}_{2}$ monolayer. (b) PL spectra of $1 \mathrm{~L}-\mathrm{WS} \mathrm{S}_{2}$ at $10 \mathrm{~K}$. Panel 1 shows a spectrum taken from a flat region away from nanopillars. Red arrows indicate unbound monolayer neutral $\left(X^{0}\right)$ and charged $\left(X^{-}\right)$excitons. Panels 2 and 3 show representative spectra of $W_{2}$ on 170 and $190 \mathrm{~nm}$ nanopillars, respectively. Insets are high-resolution PL spectra of the red-highlighted spectral regions, showing the finestructure splitting of the peaks. (c) Distribution of the emission wavelengths measured for $1 \mathrm{~L}-\mathrm{WS}_{2}$ QEs on 170 (black and white) and $190 \mathrm{~nm}$ (red) nanopillars. (d) Distribution of the number of narrow emission lines observed per nanopillar for $1 \mathrm{~L}-\mathrm{WS}_{2}$ QEs on 170 (black and white) and 190 nm (red) nanopillars. DFM, dark-field optical microscopy.

creating a single $\mathrm{QE}$ at each nanopillar site. We note that we obtain a $95 \%$ yield of $\mathrm{QE}$ creation in $1 \mathrm{~L}-\mathrm{WS}_{2}$ on non-pierced nanopillars. Further, $\sim 75 \%$ of these display two or less sub-nm emission peaks. In contrast, the 60- and the 130-nm-high nanopillars do not result in any QE occurrence (see Supplementary Fig. 7 for examples of these measurements).

\section{Discussion}

We presented a simple method for the deterministic creation of scalable arrays of quantum-light emitters embedded in LMs emitting at different regions of the optical spectrum ${ }^{33}$. The reliability of the technique will accelerate experimental studies of QEs in TMDs, which at present rely on their rather rare and random occurrence ${ }^{11-15}$. In the immediate future, a detailed study is necessary in order to achieve a better understanding of the specific role of nanopillar height and geometry in defining the characteristics of the quantum emission. We expect tunability of the optical emission by varying the shapes of the underlying nanostructures. In this respect, interesting possibilities to realise dynamical circuits using micro-electro-mechanical systems and piezoelectric tuning exist. Heterostructures may enable new routes towards tunnel-coupled quantum devices and the formation of QE molecules. Several approaches are being investigated for the production of wafer-scale samples ${ }^{34,35}$, which could lead to rapid optimization. While our approach is already compatible with standard silicon processing techniques, it is nevertheless not restricted to the specific properties of the substrate. In fact, even nanodiamonds of the appropriate dimensions, drop-cast onto silica substrates, are able to create QEs in $1 \mathrm{~L}-\mathrm{WSe}_{2}$ (see Supplementary Fig. 8 and Supplementary Note 2). The choice of substrate material will be particularly important, for example, when considering inhomogeneous linebroadening due to charge noise. Further, the flexibility in the choice of substrate, in turn, provides an opportunity to create hybrid quantum devices where LM QEs can be coupled to quantum systems in other materials such as spins in diamond and silicon carbide.

\section{Methods}

Substrate preparation. The silica nanopillar substrate is fabricated with a highresolution direct-write lithographic process via spin-on-glass polymer hydrogen silsesquioxane (HSQ) ${ }^{36}$. First, a wafer with $2 \mu \mathrm{m}$ thermal oxide is cleaved and then cleaned. HSQ resist (FOx-16, Dow-Corning) is diluted with methyl isobutyl ketone (MIBK) in different ratios and spun onto the substrate, giving variable thickness depending on the dilution. After baking at $90^{\circ} \mathrm{C}$ for $5 \mathrm{~min}$, the substrate is exposed in an electron beam lithography tool (Elionix F-125) and then developed in a $25 \%$ solution of tetramethyl ammonium hydroxide (TMAH) developer and rinsed in methanol. To convert the defined structures into pure $\mathrm{SiO}_{2}$, we apply rapid thermal annealing at $1,000^{\circ} \mathrm{C}$ in an oxygen atmosphere ${ }^{37}$, resulting in arrays of sharplydefined sub- $100 \mathrm{~nm}$ silica nanopillars.

Optical measurements. Room temperature Raman and PL measurements are carried out using a Horiba LabRam HR Evolution microspectrometer equipped with a $\times 100$ objective (numerical aperture 0.9 ) and a spot size $<1 \mu \mathrm{m}$. The pixel- 
to-pixel spectral resolution for the Raman measurements is $\sim 0.5 \mathrm{~cm}^{-1}$. Bragg gratings (BraggGrate) are used to detect the ultralow frequency Raman peaks. The power is kept below $50 \mu \mathrm{W}$ to prevent heating effects. The excitation wavelength used is $514.5 \mathrm{~nm}$ for $\mathrm{WSe}_{2}$ and $457 \mathrm{~nm}$ for $\mathrm{WS}_{2}$.

A variable-temperature helium flow cryostat (Oxford Instruments Microstat HiRes2) is used to perform low-temperature PL measurements with a home-built confocal microscope mounted on a three-axis stage (Physik Instrumente $\mathrm{M}-405 \mathrm{DG})$ with a 5-cm travel range, 200-nm resolution for coarse alignment and a piezo scanning mirror (Physik Instrumente S-334) for high-resolution raster scans. $\mathrm{PL}$ is collected using a $1.7-\mathrm{mm}$ working distance objective with a numerical aperture of 0.7 (Nikon S Plan Fluor $\times 60$ ) and detected on a fibre-coupled singlephoton-counting module (PerkinElmer: SPCM-AQRH). Photon correlations from a Hanbury Brown and Twiss interferometer are recorded with a time-to-digital converter (quTAU). A double grating spectrometer (Princeton Instruments) is used for acquiring spectra. For PL measurements, the excitation laser $(532 \mathrm{~nm}$, Laser Quantum) is suppressed with a long pass filter (550 nm Thorlabs FEL0550).

Data availability. The data that supports the findings of this study are available from the corresponding author upon request.

\section{References}

1. Cao, T. et al. Valley-selective circular dichroism of monolayer molybdenum disulphide. Nat. Commun. 3, 887 (2012).

2. Zeng, H., Dai, J., Yao, W., Xiao, D. \& Cui, X. Valley polarization in $\mathrm{MoS}_{2}$ monolayers by optical pumping. Nat. Nanotechnol. 7, 490-493 (2012).

3. Mak, K. F., He, K., Shan, J. \& Heinz, T. F. Control of valley polarization in monolayer $\mathrm{MoS}_{2}$ by optical helicity. Nat. Nanotechnol. 7, 494-498 (2012).

4. Mak, K. F. et al. Tightly bound trions in monolayer $\mathrm{MoS}_{2}$. Nat. Mater. 12, 207-211 (2013).

5. He, K. et al. Tightly bound excitons in monolayer $\mathrm{WSe}_{2}$. Phys. Rev. Lett. 113, 26803 (2014).

6. Sidler, M. et al. Fermi polaron-polaritons in charge-tunable atomically thin semiconductors. Nat. Phys. 13, 255-261 (2016).

7. Bonaccorso, F. et al. Production and processing of graphene and $2 \mathrm{~d}$ crystals. Mater. Today 15, 564-589 (2012).

8. Akinwande, D., Petrone, N. \& Hone, J. Two-dimensional flexible nanoelectronics. Nat. Commun. 5, 5678 (2014).

9. Majumdar, A. et al. Hybrid 2D material nanophotonics: a scalable platform for low-power nonlinear and quantum optics. ACS Photonics 2, 1160-1166 (2015).

10. Xia, F., Wang, H., Xiao, D., Dubey, M. \& Ramasubramaniam, A. Twodimensional material nanophotonics. Nat. Photonics 8, 899-907 (2014).

11. Tonndorf, P. et al. Single-photon emission from localized excitons in an atomically thin semiconductor. Optica 2, 347 (2015).

12. Srivastava, A. et al. Optically active quantum dots in monolayer $\mathrm{WSe}_{2}$. Nat. Nanotechnol. 10, 491-496 (2015).

13. He, Y.-M. et al. Single quantum emitters in monolayer semiconductors. Nat. Nanotechnol. 10, 497-502 (2015).

14. Koperski, M. et al. Single photon emitters in exfoliated $\mathrm{WSe}_{2}$ structures. Nat. Nanotechnol. 10, 503-506 (2015).

15. Chakraborty, C., Kinnischtzke, L., Goodfellow, K. M., Beams, R. \& Vamivakas, A. N. Voltage-controlled quantum light from an atomically thin semiconductor. Nat. Nanotechnol. 10, 507-511 (2015).

16. Tran, T. T., Bray, K., Ford, M. J., Toth, M. \& Aharonovich, I. Quantum emission from hexagonal boron nitride monolayers. Nat. Nanotechnol. 11, 37-41 (2015).

17. Bonaccorso, F., Sun, Z., Hasan, T. \& Ferrari, A. C. Graphene photonics and optoelectronics. Nat. Photonics 4, 611-622 (2010).

18. Koppens, F. H. L. et al. Photodetectors based on graphene, other twodimensional materials and hybrid systems. Nat. Nanotechnol. 9, 780-793 (2014).

19. Ferrari, A. C. Science and technology roadmap for graphene, related two-dimensional crystals, and hybrid systems. Nanoscale 7, 4598-4810 (2015).

20. Aharonovich, I., Englund, D. \& Toth, M. Solid-state single-photon emitters. Nat. Photonics 10, 631-641 (2016).

21. Branny, A. et al. Discrete quantum dot like emitters in monolayer $\mathrm{MoSe}_{2}$ : spatial mapping, magneto-optics, and charge tuning. Appl. Phys. Lett. 108, 142101 (2016)

22. Palacios-Berraquero, C. et al. Atomically thin quantum light-emitting diodes Nat. Commun. 7, 12978 (2016).

23. Kumar, S., Kaczmarczyk, A. \& Gerardot, B. D. Strain-induced spatial and spectral isolation of quantum emitters in mono- and bilayer $\mathrm{WSe}_{2}$. Nano Lett. 15, 7567-7573 (2015).

24. Kern, J. et al. Nanoscale positioning of single-photon emitters in atomically thin $\mathrm{WSe}_{2}$. Adv. Mater. 28, 7101-7105 (2016).
25. Novoselov, K. S. et al. Two-dimensional atomic crystals. Proc. Natl Acad. Sci. USA 102, 10451-10453 (2005).

26. Casiraghi, C. et al. Rayleigh imaging of graphene and graphene layers. Nano Lett. 7, 2711-2717 (2007).

27. Castellanos-Gomez, A. et al. Deterministic transfer of two-dimensional materials by all-dry viscoelastic stamping. 2D Mater. 1, 11002 (2014).

28. Terrones, H. et al. New first order Raman-active modes in few layered transition metal dichalcogenides. Sci. Rep. 4, 4215 (2014).

29. Zhao, W. et al. Lattice dynamics in mono- and few-layer sheets of $\mathrm{WS}_{2}$ and $\mathrm{WSe}_{2}$. Nanoscale 5, 9677-9683 (2013).

30. Zhou, B. et al. Evolution of electronic structure in atomically thin sheets of $\mathrm{WS}_{2}$ and WSe $\mathrm{W}_{2}$. ACS Nano 7, 791-797 (2013).

31. Kumar, S. et al. Resonant laser spectroscopy of localized excitons in monolayer $\mathrm{WSe}_{2}$. Optica 3, 882 (2016).

32. Wang, G. et al. Valley dynamics probed through charged and neutral exciton emission in monolayer $\mathrm{WSe}_{2}$. Phys. Rev. B 90, 075413 (2014).

33. Jiang, M., Kurvits, J. A., Lu, Y., Nurmikko, A. V. \& Zia, R. Reusable inorganic templates for electrostatic self-assembly of individual quantum dots, nanodiamonds, and lanthanide-doped nanoparticles. Nano Lett. 15, 5010-5016 (2015).

34. Kang, K. et al. High-mobility three-atom-thick semiconducting films with wafer-scale homogeneity. Nature 520, 656-660 (2015).

35. Ling, X. et al. Parallel stitching of 2D Materials. Adv. Mater. 28, 2322-2329 (2016).

36. Grigorescu, A. E. et al. Resists for sub-20-nm electron beam lithography with a focus on HSQ: state of the art. Nanotechnology 20, 292001 (2009).

37. Holzwarth, C. W., Barwicz, T. \& Smith, H. I. Optimization of hydrogen silsesquioxane for photonic applications. J. Vac. Sci. Technol. B Microelectron. Nanometer Struct. Process. Meas. Phenom. 25, 2658 (2007).

\section{Acknowledgements}

We acknowledge financial support from the Marie Skłodowska-Curie Actions SpinNANO, Grant No. 676108, EU Graphene Flagship, ERC Grants Hetero2D and PHOENICS, EPSRC Grants EP/K01711X/1, EP/K017144/1, EP/N010345/1, EP/M507799/1, EP/L016087/1, Quantum Technology Hub NQIT EP/M013243/1, the EPSRC Cambridge NanoDTC, Graphene Technology CDT, EP/G037221/1 and the STC Center for Integrated Quantum Materials (NSF Grant No. DMR-1231319). We thank H.S. Knowles and P. Borisova for technical assistance.

\section{Author contributions}

M.A., A.C.F. and M.L. managed the project. M.A., D.M.K. and C.P.-B. devised the project. P.L. fabricated the nanopatterned substrates. C.P.-B., A.R.-P.M. and M.B. developed the deposition method and fabricated the samples. C.P.-B., A.R.-P.M., D.M.K., A.K.O., M.B. and D.Y. performed the optical measurements. C.P.-B., A.R.-P.M. and M.B performed AFM measurements. All authors participated in the results discussions, analysis and the writing of the manuscript.

\section{Additional information}

Supplementary Information accompanies this paper at http://www.nature.com/ naturecommunications

Competing interests: The authors declare no competing financial interests.

Reprints and permission information is available online at http://npg.nature.com/ reprintsandpermissions/

How to cite this article: Palacios-Berraquero, C. et al. Large-scale quantum-emitter arrays in atomically thin semiconductors. Nat. Commun. 8, 15093 doi: $10.1038 /$ ncomms15093 (2017)

Publisher's note: Springer Nature remains neutral with regard to jurisdictional claims in published maps and institutional affiliations.

This work is licensed under a Creative Commons Attribution 4.0 International License. The images or other third party material in this article are included in the article's Creative Commons license, unless indicated otherwise in the credit line; if the material is not included under the Creative Commons license, users will need to obtain permission from the license holder to reproduce the material. To view a copy of this license, visit http://creativecommons.org/licenses/by/4.0/

(C) The Author(s) 2017 